\title{
Prevalence and Antimicrobial Susceptibility Pattern of Methicillin Resistance Staphylococcus in a Sudanese Surgical Ward*
}

\author{
Salah Ibrahim Kheder ${ }^{1 \#}$, Nagla A. Ali ${ }^{2}$, Ahmed Ibrahim Fathelrahman ${ }^{3,4}$ \\ ${ }^{1}$ Pharmacy Program, National College of Medical \& Technical Sciences, Khartoum, Sudan; ${ }^{2}$ Department of Microbiology, Faculty of \\ Medicine \& Health Sciences, Omdurman Islamic University, Khartoum, Sudan; ${ }^{3}$ Ministry of Health, Khartoum State, Khartoum, \\ Sudan; ${ }^{4}$ Department of Pharmacy Practice, College of Pharmacy, Qassim University, Buraidah, Qassim, Saudi Arabia. \\ Email: \#isra-su@hotmail.com
}

Received October $27^{\text {th }}, 2011$; revised November $11^{\text {th }}, 2011$; accepted December $20^{\text {th }}, 2011$

\begin{abstract}
Background: Methicillin-resistant Staphylococcus aureus (MRSA) is important nosocomial pathogen. Aim: In this paper, we determined the prevalence and the antibiotic susceptibility pattern of (MRSA) in a Sudanese surgical ward. Method: A total of 200 post-operative surgical specimens were collected from patients hospitalized in gastrointestinal tract (GIT) surgical ward in Ibn Sina hospital, Khartoum, Sudan and were subjected to MRSA screening and sensitivity test. Key Findings: Out of 35 strains of Staphylococcus aureus isolated from surgical samples, 25 (71.4\%) were found to be MRSA. Almost all MRSA strains were resistant to Methicillin, $96 \%$ to Ofloxacin, $92 \%$ to Pencillin G, $24 \%$ to Amikacin and 4\% to Vancomycin. Cross-resistance was obviously detected. Conclusion: The present study detected alarming levels of $S$. aureus (MRSA) isolates, at the same time presence of high cross-resistance to other antibiotics.
\end{abstract}

Keywords: Methicillin-Resistant Staphylococcus aureus; Sudanese Hospitals; Antimicrobial Sensitivity; Infection Control; Antibiotic Policy

\section{Introduction}

Staphylococcus aureus has been reported as a major cause of community and hospital acquired infections [1]. Methicillin-resistant Staphylococcus aureus (MRSA) are bacteria resistant to penicillin and cephalosporin classes of antibiotics and are often resistant to many other classes of antibiotics [2]. MRSA strains were initially described in 1961 and emerged in the last decade as one of the most important noscomial pathogens which was reported just one year after the launch of methicillin [3]. In MRSA, the horizontally acquired mecA gene encodes a penicillin-binding protein (PBP2a), which is intrinsically insensitive to methicillin and all $\beta$-lactams that have been developed, including the isoxazoyl penicillins (e.g. Oxacillin) that superceded methicillin [4].

MRSA infections are a problem across the whole health economy, and have been shown to be associated with a poorer outcome and higher mortality than similar infections caused by methicllin-sensitive strains of $S$. aureus $[5,6]$. Many of these isolates are becoming mul-

${ }^{*}$ Conflict of Interest Statement: We declare that we have no conflict of interest.

${ }^{\#}$ Corresponding author. tidrug resistant and are susceptible only to glycopeptides antibiotics such as vancomycin [7]. The prolonged hospital stay, poor infection control measures, indiscriminate, irregular use of antibiotics, lack of awareness, receipt of antibiotics before coming to hospitals are some of the possible predisposing factors of MRSA emergence and transmission [8]. Fighting MRSA involves re-enforcement of infection control measures as well as rational use of antimicrobials. Therefore, the knowledge of the prevalence of MRSA and screening of surgical patients for MRSA, in addition to identifying their current antimicrobial profile become necessary to facilitate appropriate selection of antibiotic agents for pre and postoperative prophylaxis [9]. However, robust evidence of which control measures are most effective remains largely elusive. Hand hygiene is also believed to be crucial, but it is effective long-term implementation has proved to be problematic $[10,11]$. In the current study, we determined the prevalence of MRSA from surgical swab samples and their in-vitro susceptibility pattern to various antimicrobial classes, and we also evaluated the effect of infection control and antibiotic use interventions on MRSA rates in gastro-intestinal (GIT) surgical ward in Ibn Sina hospital. 


\section{Methods}

\subsection{Samples, Setting and Study Period}

Patients underwent surgical operation and treated empirically with antibiotics for more than 48 hours then developed post-operative infections in GIT surgical ward, in Ibn Sina hospital were consecutively enrolled into study, and followed prospectively. Ibn Sina hospital is a 132-bed specialized secondary hospital located in Khartoum the main city and the capital of Sudan. The GIT surgical ward is a 52-bed ward. All the microbiological testing was performed in the hospital laboratory and laboratory data records were collected and analyzed for the period from 1 May 2008 to 30 August 2009. Specimens of pus collected from the surgical wounds in sterile swabs and cultured on conventional media of Nutrient agar, and Mannitol salt agar. In case of duplicate isolates, the second isolate was excluded from the antibiogram reporting. Duplicate isolates are defined as isolates of the same organism obtained from the same patient within pre-specified period of time. During this period an ongoing intervention study to investigate the impact of quaternary antimicrobial cycling policy on antimicrobial resistance and utilization was conducted, and this period coincide with the baseline and first three cyclic periods $[12,13]$. Also during the first cyclic period alcohol-based hand rub dispensers had been introduced. The study approval was obtained from hospital administration.

\subsection{Susceptibility Testing}

The morphotypes were done for all samples based on the Gram staining method to determine the most likely organism present. Organisms were identified according to standard procedures [14]. Susceptibility testing was carried out by disc diffusion method following the National Committee for Clinical Laboratory Standards (NCCLS) guidelines [15]. To differentiate between Staphylococcus aureus and other staphylococci organisms coagulase test was done (Coagulase positive confirm S. aureus). All the confirmed Staphylococcus aureus strains were subse- quently tested for methicillin resistance by screening for susceptibility to oxacillin. The isolates were labeled as MRSA if it was observed to be resistant to oxacillin disc after incubation at $37^{\circ} \mathrm{C}$ for 24 hours (Oxacillin-resistance on laboratory susceptibility testing also indicates methicillin-resistance) [15].

\subsection{Cross-Resistance Profile}

To carefully characterize the patterns of resistance of MRSA, cross resistance of isolates to 5 antibiotics with different mechanisms of action were detected. Those include Methicillin, Amikicin, Vancomycin, Penicillin G and Ofloxacin.

\subsection{Study Outcomes}

The primary outcome was the prevalence and susceptibility pattern of MRSA isolates. Secondary outcome was the impact of antimicrobial cycling policy and hand hygiene infection control on MRSA susceptibility and cross-resistance.

\subsection{Data Processing and Analysis}

Data collected was processed and analyzed using WHONET software. Analysis was performed under the condition as one isolate per one patient. (WHONET is data-base software which established and implemented by World Health Organization as a surveillance tool for antimicrobial resistant) [16].

\section{Results}

During study period, surgical swabs samples were obtained from 200 patients. Of those, 104 (52\%) were male. On culture, 35 samples were confirmed to be Staphylococcus aureus strains. All these were tested for methicillin resistance and 25 (71.4\%) were found to be MRSA, whereas 10 (28.6\%) were methicillin sensitive $S$. aureus (MSSA). The 35 samples were tested for sensitivity to additional antibiotics as shown in Table 1.

Table 1. Antimicrobial susceptibility patterns of $S$. aureus $(\mathbf{n}=35)$.

\begin{tabular}{|c|c|c|c|c|}
\hline Antibiotic name & Antibiotic class & Resistant Frequency (\%) & Sensitive Frequency (\%) & \% R 95\% C.I \\
\hline Oxacillin & Penicillins & $25(71.4 \%)$ & $10(28.6 \%)$ & $53.4-84.7$ \\
\hline Methicillin & Penicillins & 27 (77\%) & $8(23 \%)$ & $57.6-86.1$ \\
\hline Penicillin G & Penicillins & 33 (94\%) & $2(6 \%)$ & $82.4-99.1$ \\
\hline Amikacin & Aminoglycosides & $6(17 \%)$ & $29(83 \%)$ & $7.1-34.3$ \\
\hline Ofloxacin & Quinolones & $30(86 \%)$ & $5(14 \%)$ & $68.9-94.6$ \\
\hline Vancomycin & Glycopeptides & $2(6 \%)$ & 33 (94\%) & $1.0-20.5$ \\
\hline
\end{tabular}

\% R = Resistance \%; 95\% C.I = 96\% Confidence Intervals. 


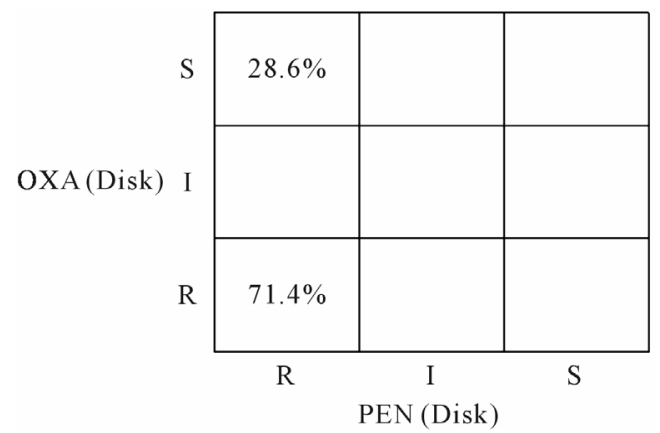

Figure 1. Scatterplot which compare sensitivity test for $S$. aureus to oxacillin and penicillin antibiotics. OXA: Oxacillin, PEN: Penicillin G, R: resistant, S: sensitive.

The drug resistance patterns of MRSA isolated from surgical and wound specimens was found to be highly variable. Almost all of the 25 oxacillin resistant strains (MRSA) were resistant to Methicillin, 96\% to Ofloxacin, 92\% to Pencillin, 24\% to Amikacin, and 4\% to Vancomycin.

Scatterplot figures from 1 - 3 compared oxacillin disc resistant phenotypes with penicillin, oflaxacin and vancomycin discs.

As shown in Figure 1, 71.4\% of isolates were in the lower left-hand quarter representing the proportion that was resistant to both antibiotics, oxacillin and penicillin G; while $28.6 \%$ were in the upper left-hand quarter of the graph representing the proportion that was resistant to penicillin but sensitive to oxacillin

In Figure 2, 68.6\% of isolates were in the lower lefthand quarter representing the proportion that was resistant to both antibiotics, oxacillin and ofloxacin; $2.9 \%$ were in the lower right-hand quarter representing the proportion that was resistant to oxacillin but sensitive to ofloxacin, $11.4 \%$ in the upper right-hand quarter of the graph (sensitive to both oxacillin and ofloxacin), and $17.1 \%$ of the isolates were in the upper-left hand quarter of the graph (sensitive to oxacillin but resistant to ofloxacin).

In Figure 3, only 2.9\% of isolates were in the lowerleft hand quarter, representing the proportion that was resistant to both antibiotics, oxacillin and vancomycin; $68.6 \%$ of isolates were in the lower right-hand quarter (resistant to oxacillin but sensitive to vancomycin), whereas $25.7 \%$ of isolates were in the upper left-hand quarter (sensitive to both antibiotics), and $2.9 \%$ of the isolates were in the upper-left hand quarter of the graph (sensitive to oxacillin but resistant to vancomycin).

Figure 4 addresses the impact of antimicrobial cycling policy and hand hygiene infection control measures on the MRSA \%. A pronounced reduction in resistance to all antibiotics used were observed in cycle 1 (Cepalosporins cycle) when compared to baseline period and

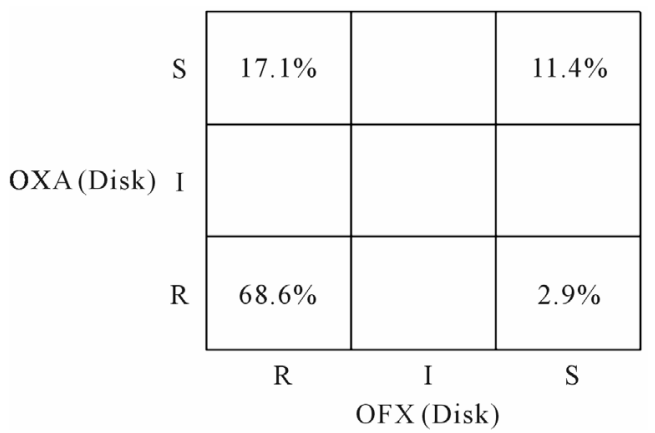

Figure 2. Scatterplot which compare sensitivity test for $S$. aureus to oxacillin and ofloxacin antibiotics. OXA: Oxacillin, OFX: Ofloxacin, R: resistant, S: sensitive.

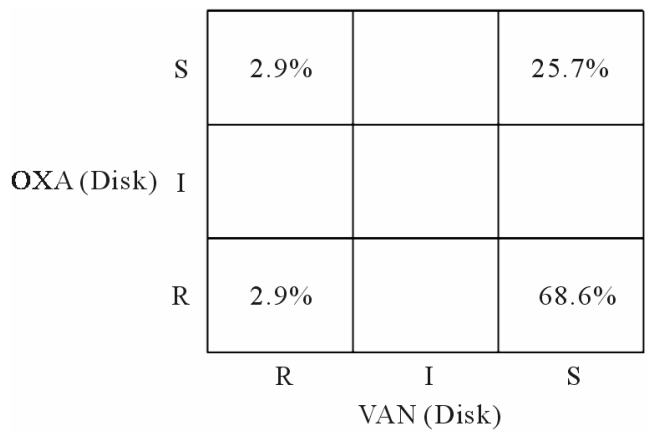

Figure 3. Scatterplot which compare sensitivity test for $S$. aureus to oxacillin and vancomycin antibiotics. OXA: Oxacillin, VAN: Vancomycin, R: resistant, S: sensitive.

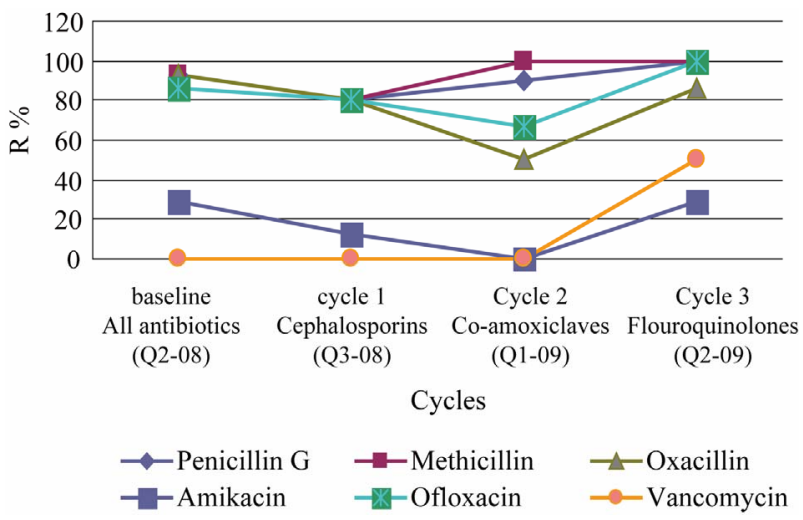

Figure 4. Percentage of MRSA resistance to antibiotic per cyclic periods. $(\mathrm{Q} 2-08$ = May-Aug 2008, Q3-08 $=$ Sept-Dec 2008, Q1-09 = Jan-Apr 2009, Q2-09 = May-Aug 2009).

increased to two antibiotics (methicillin and penicillin G) in cycle 2 (Co-amoxiclaves cycle), while it increased again for all antibiotics used in cycle 3 (Flouroquinolones cycle).

\section{Discussion}

This study was designed to determine the prevalence of MRSA isolates among Staphylococcus aureus isolated 
and screened from GIT surgical ward in Ibn Sina hospital. Alarming levels of MRSA isolates were detected (12.5\% was the prevalence rate of $S$. aureus, and $71.4 \%$ of them were MRSA). Similar prevalence rate of MRSA (74\%) was observed in another study conducted in 2007 to determine prevalence of antimicrobial resistance among pathogenic bacteria isolated from three major hospitals in Khartoum (Khartoum teaching hospital, Ear Nose \& Throat teaching hospital ,and Omdurman teaching hospital) [17]. Levels reported elsewhere differed substantially ranging from $22 \%-65 \%[18,19]$. However, there are evidences that the prevalence of MRSA has increased worldwide [20-22].

The majority of the MRSA isolates were resistant to multiple antimicrobial agents, as previously noted in the literature [21]. In the present study out of 25 MRSA strains isolated, average of $63 \%$ exhibited high resistance to additional 5 antibiotics tested. These MRSA were multi drug resistant Staphylococcus aureus. Hence, multi-resistance strains might be overwhelmingly dominant in the ward of surgery, as indicated by the persistent multi-resistance profile among all cyclic periods.

The absence of such a rapid decrease in resistance was, at least partly, the result of cross-resistance to multiple antibiotics, where an antibiotic belonging to one class may become prone to resistance that is commonly known for drugs belonging to one or more unrelated classes. This is a result of genetic linkage of resistance determinants that encode resistance to multiple classes of antibiotics. This can be clearly explained by scatteplot graphs which is useful in both description of molecular mechanisms of resistance as well as in detection of the unusual or impossible phenotypes suggestive of possible errors (i.e. such comparisons is useful in studying mechanisms of resistance and quality assurance by finding unusual resistance phenotype. $28.6 \%$ of the isolates were resistant to penicillin but sensitive to oxacillin. This is classical phenotype for Beta-lactamase (pencillinase producing) S.aureus. Fortunately; there is no isolates would have the phenotype that was penicillin-sensitive and oxacillinresistant. Microbiologically such isolates are exceedingly rare, and most likely attributable to an error in the laboratory (poor quality reagent, test performance, measurements, etc). Although the antibiotics oxacillin and ofloxacin (similarly ciprofloxacin) are from different classes of antimicrobials with distinct mechanisms of resistance, they were subjected to cross resistance between each other indicating that genetic linkage between classes is commonly observed. About $70 \%$ of isolates were sensitive to vancomycin but resistant to oxacillin and about $3 \%$ isolate are resistant to both. Such finding indicates the emergence of an extended resistance from MRSA to vancomycin resistance Staphylococcus aureus (VRSA).
From scatterplot graphs, we can conclude that there is high cross-resistance between antibiotics and also multiresistance strains carried by most of the patient admitted to surgical ward. This makes the empirical use of antibacterial effective against MRSA imperative and left us with a few alternative choices such as vancomycin and linezolid. Once introduced, the eradication becomes difficult as vancomycin is costly and linezolid not available in our country till now.

During the present study, an antibiotic rotation policy study implemented and an alcohol-based hand hygiene introduced as infection control measures in whole hospital may be a confounding factors for high drop of rate of prevalence of colonization from baseline to cycle 2, but it increased in cycle 3. This suggests that infection control campaign was enforced in the first cyclic periods 1 \& 2 but it seems remained not identical and less stringent for the remainder of the study period as the rate of resistance increased in cycle 3, especially that poor and inconsistent compliance had been approved in the same hospital [23]. Also the high use of cephalosporin has been associated with an increased prevalence of MRSA and vancomycin-resistant enterocooci (VRE), although a direct causal relationship has not been established [24]. The reduction in antibiotic resistant organisms and infections may be due to the antibiotic rotation or other factors. However, many studies confirmed that altering policy or antibiotic rotation potentially decreases the incidence of infection with both antibiotic sensitive and antibiotic resistant bacteria [25-27].

\section{Conclusion}

The present study detected alarming levels of S.aureus (MRSA) isolates, at the same time presence of high cross-resistance to other antibiotics. Constant monitoring of these strains is essential in order to control their spread in the hospital environment and transmission to the community. In addition, this is essentially important to facilitate appropriate selection of antibiotic agents by clinicians. Intervention such as antibiotic prescribing polices and stringent infection control measures are needed to be enforced.

\section{REFERENCES}

[1] J. N. Sheagren, "Staphylococcus aureus. The Persistent Pathogen,” New England Journal of Medicine, Vol. 310, 1984, pp. 1368-1373. doi:10.1056/NEJM198405243102107

[2] A. McGeer, et al., "Defintions of Infection for Surveillance in Long-Term Care Facilities," American Journal of Infection Control, Vol. 19, No. 1, 1991, pp. 1-7. doi:10.1016/0196-6553(91)90154-5 
[3] P. A. C. Maple, J. M. T. Hamilton-Miller and W. Brumfitt, "Worldwide Antibiotic Resistance in Methicillin Resistant Staphylococcus aureus," Lancet, Vol. 1, 1989, pp. 537-540. doi:10.1016/S0140-6736(89)90076-7

[4] T. J. Foster, "The Staphylococcus aureus (Super Bug)," Journal of clinical Investigation, Vol. 114, No. 12, 2004, pp. 1693-1696.

[5] S. E. Cosgrove, G. Skoulas, E. N. Perencevish, M. J. Schwaber, A. W. Karchmer and Y. Carmeli, "Comparison of Mortality Associated with Methicillin-Resistant and Methicillin-Susceptible Staphylococcus aureus Bactermia: A Meta-Analysis," Clinical Infectious Diseases, Vol. 36, No. 1, 2003, pp. 53-59. doi:10.1086/345476

[6] M. Whitby, M. L. McLaws and G. Berry, "Risk of Death from Methicillin-Resistant Staphylococcus aureus Bacteremia: A Meta-Analysis,” Medical Journal of Australia, Vol. 175, 2001, pp. 264-267.

[7] A. P. Mehta, C. Rodrigues, K. Sheth, S. Jani, A. Hakimiyan and N. Fazalbhoy, "Control of Methicillin-Resistant Staphylococcus aureus in a Tertiary Care Centre. A FiveYear Study,” Journal of Medical Microbiology, Vol. 16, 1998, pp. 31-34.

[8] S. Anupurba, M. R. Sen, G. Nath, B. M. Sharma, A. K. Gulati and T. M. Mohapatra, "Prevelance of Methicillin-Resistant Staphylococcus aureus in a Tertiary Care Referral Hospital in Eastern Uttar Pradesh,” Indian Journal of Medical Microbiology, Vol. 21, No. 1, 2003, pp. 49-51.

[9] G. Zanetti, S. J. Goldie and R. Platt, "Clinical Consequencies and Cost of Limiting Use of Vancomycin for Preoperative Prophylaxis: Example of Coronary Artery Bypass Surgery," Emerging Infectious Diseases, Vol. 7, No. 5, 2001, pp. 820-827. doi:10.3201/eid0705.010508

[10] C. Marshal, S. Wesselingh, M. McDonald and D. Spelman, "Control of Endemic MRSA - What Is the Evidence? A Personal Review,” Journal of Hospital Infection, Vol. 56, No. 4, 2004, pp. 253-268.

[11] H. Grundmann, M. Aires-de-Sousa, J. Boyce and E. Tiemersma, "Emergence and Resurgence of MethicillinResistant Staphylococcus aureus as a Public-Health Threat,” Lancet, Vol. 368, No. 9538, 2006, pp. 874-885. doi:10.1016/S0140-6736(06)68853-3

[12] S. I. Kheder, I. Eltayeb, S. A. I. Shaddad and E. Kheder, "Impact of Antibiotic Cycling Policy in Antimicrobial Resistance in, Two Sudanese Surgical Wards Settings: Prospective Longitudinal Interventional Study,” Journal of Pharmaceutical and Biomedical Sciences, Vol. 5, No. 7, 2011, pp. 1-10.

[13] S. I. Kheder, "Antibiotic Utilization and Prescribers Adherence Measurements in a Sudanese Hospital Settings after Introducing Antibiotic Policy," Sudan Medical Monitor, Vol. 6, No. 1, 2011, pp. 39-48.

[14] M. Cheesbrough, "District Laboratory Practice in Tropical Countries Part (2),” Cambridge University Press, Cambridge, 1999.

[15] National Committee for Clinical Laboratory Standards, "Performance Standards for Antimicrobial Disc Suscepti- bility Tests, Approved Standard M2-A6,” 6th Edition, NCCLS, Wayne, 1999.

[16] J. M. Stelling and T. F. O’breien, "Suroveillance of Antimicrobial Resistance: The WHONET Program," Clinical Infectious Diseases, Vol. 24, Suppl. 1, 1997, pp. S157S168. doi:10.1093/clinids/24.Supplement_1.S157

[17] M. A. Alsadig, "Resistance of Pathogenic Bacteria to Antibiotics in Use in Hospitals and Community," Council of Biological Sciences, New Technologies and Environment Tropical Medicine Programmers' Khartoum, Sudan Academy of Sciences, SAS, 2007.

[18] N. Pal and A. Ayyagari, "Drug Resistance Pattern of Methicillin-Resistant Staphylococcus aureus," Indian Pediatrics, Vol. 28, 1991, pp. 731-733.

[19] M. Borg, E. Scicluna, M. De Kraker, N. Van de Sande, E. Tiemersma, D. Gur, S. Ben Redjeb, O. Rasslan, Z. Elnassar, M. Benbachir, D. Pieridu Bagatzouni, K. Rahal, Z. Daoud, H. Grundmann and J. Monen, “Antibiotic Resistance in the Southeastern Mediterranean. Preliminary Results from AR Medical Project,” European Surveillance, Vol. 11, No. 7, 2006, pp. 164-167.

[20] D. J. Diekema, M. A. Pfaller, F. J. Schmitz, J. Smayersky, J. Bell, R. N. Jones, M. Beech and Sentry Partcipant Group, "Survey of Infections Due to Staphylococci Species: Frequency of Occurrence and Antimicrobial Susceptibility of Isolates Collected in the United States, Canada, Latin America, Europe, and the Western Pacific Region for the Sentry Antimicrobial Surveillance Program, 19971999,” Clinical Infectious Disease, Vol. 32, Suppl. 2, 2001, pp. S114-S132. doi:10.1086/320184

[21] A. C. Fluit, C. L. Wielders, J. Verhoef and F.J. Schmitz, "Epidemiology and Susceptibility of 3051 S. aureus Isolates from 25 University Hospitals Participating in the Europen Sentry Study," Journal of Clinical Microbiology, Vol. 39, 2001, pp. 3727-3732. doi:10.1128/JCM.39.10.3727-3732.2001

[22] W. Witte, “Antibiotic Resistance in Gram-Positive Bacteria: Epidemiological Aspects,” Journal of Antimicrobial. Chemotherapy, Vol. 44, Suppl. 1, 1999, pp. 1-9. doi:10.1093/jac/44.suppl_1.1

[23] S. I. Kheder, I. Eltayeb and S. A. I. Shaddad, "Evaluation of Hand Hygiene Adherence by Health Care Workers in a Secondary Sudanese Hospital Setting," Sudan Medical Monitor, Vol. 6, No. 1, 2011, pp. 7-12.

[24] H. S. Gold and R. C. Moellering, “Antimicrobial-Drug Resistance,” New England Journal of Medicine, Vol. 335, 1996, pp. 1445-1453. doi:10.1056/NEJM199611073351907

[25] H. Badawi, M. S. Diab and M. Elsaid, "Impact of Antibiotic Policy in a Tertiary Care Research Institute Hospital in Egypt: Three Years Experience,” International Journal of Infection Control, Vol. 3, No. 2, 2007, pp. 1-7. doi:10.3396/03-01-10-07

[26] M. G. Hughes, H. L. Evans, T. W. Chong, R. L. Smith and R. G. Sawyer, "Effect of an Intensive Care Rotating Empiric Antibiotic Schedule on the Development of Hospital-Acquired Infections on the Non-Intensive Care Unit Ward," Critical Care Medicine, Vol. 32, No. 1, 2004, pp. 
53-60. doi:10.1097/01.CCM.0000104463.55423.EF

[27] S. I. Kheder, I. Eltayeb, S. A. I. Shaddad and E. Kheder, "Effect of Antibiotic Rotation Protocol on the Develop- ment of Hospital Acquired Infections in Hospital Surgical Units in Sudan,” Sudan Medical Monitor, Vol. 5, No. 4, 2010, pp. 165-173. 